\title{
Real-Life-Daten
}

\section{Eisenmangeltherapie: Der Patient im Fokus}

Patienten mit Eisenmangel und Grund- oder Begleiterkrankungen können von einer Behandlung mit i.v. Eisencarboxymaltose hinsichtlich Symptomatik und Lebensqualität profitieren. Dies zeigen die Ergebnisse einer aktuellen nicht-interventionellen Real-Life-Studie.

Eingeschlossen in die prospektive, nicht-interventionelle Studie wurden 936 Patienten mit Eisenmangel und unter-

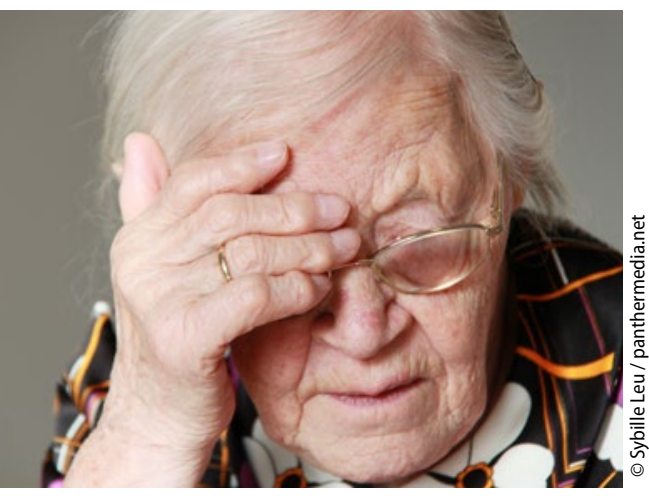

Schwäche - ein frühes Symptom der Anämie. schiedlichen Grund- oder Begleiterkrankungen in 165 allgemeinmedizinischen Zentren. Ausschlusskriterien waren stark ausgeprägte Komorbiditäten sowie eine Vorbehandlung mit i.v.-Eisenpräparaten oder Erythropoetin (EPO) bis sechs Wochen vor Studienbeginn oder mit Transfusionen bis sieben Tage vor Studienbeginn. 907 Patienten erhielten i.v. eine Initialdosis von 1.000 mgEisencarboxymaltose (ferinject $\left.{ }^{\star}\right)$. Das Ende des Beäbachtungszeitraums lag bei 12 Wochen.

\section{Gewinn an Lebensqualität}

Zum Studienende hatte sich der Status der Grunderkrankungen aus Sicht der Ärzte bei Patienten mit chronischer Herzinsuffizienz in $46,8 \%$, mit Niereninsuffizienz in $23,7 \%$ und mit gastrointestinalen Erkrankungen in 59,3\% der Fälle verbessert. Die Lebensqualität wurde mittels der FACIT Fatigue Scale bei 703 Patienten, die ein Tagebuch geführt hatten, ermittelt. Nach 12 Wochen nahm der FACIT-Summenscore signifi- kant von 22,6 auf 40,6 Punkte zu (p < 0,0001 ).

\section{Hohe Therapiezufriedenheit}

Die Ärzte bewerteten den Einfluss der Therapie auf Symptome, Leistungsfähigkeit, Eisenindizes und Verträglichkeit mit Noten um 3,5 auf einer Skala von 0 (unzufrieden) bis 4 (sehr zufrieden). Rund $80 \%$ der Patienten waren mit der Therapie bezüglich Leistungsfähigkeit und körperlicher Symptomatik zufrieden bzw. sehr zufrieden. Über 90\% der Ärzte und Patienten würden i.v. Eisencarboxymaltose erneut anwenden. „Damit bestätigt die Real-Life-Studie die Resultate aktueller klinischer Studien, in denen sich diese Behandlungsmethode als wirksam und sicher erwiesen hat", resümierte Studienleiter Dr. Viktor Habermehl, Tönisvorst.

Gudrun Girrbach

- Symposium „Eisentherapie - ein neuer Wirkansatz in der Herzinsuffizienz", 122. Kongress der Deutschen Gesellschaft für Innere Medizin e.V. (DGIM); Mannheim, April 2016 (Veranstalter: Vifor)

\section{Chronisch entzündliche Darmerkrankungen \\ Keine Angst vor Schwangerschaft}

_Die chronisch entzündlichen Darmerkrankungen (CED) manifestieren sich vorwiegend im jungen Erwachsenenalter. Das ist ein Alter, in dem für betroffene Patienten das Thema Familienplanung eine wichtige Rolle spielt. „Dabei ist die Angst weit verbreitet, dass die medikamentöse Therapie bei Mutter oder Vater das Missbildungsrisiko erhöhen könnte", erläuterte PD Dr. Niels Teich, Leipzig.

Auch befürchten Betroffene, dass die Erkrankung selbst vererbt werden könnte. Dagegen machen sich die Patienten weniger Sorgen darüber, dass die Schwangerschaft ihren Krankheitsverlauf negativ beeinflussen könnte. Was die Fertilität betrifft, so ist diese nur im akuten Schub oder bei einer chronischen Eisenmangelanämie mit
Amenorrhoe, einer Pouch-Anlage oder Abszessen bzw. Fisteln beeinträchtigt.

\section{Hohe Krankheitsaktivität ist ein Risiko}

„Bezüglich der Teratogenität der eingesetzten Medikamente gibt es bei Frauen keinerlei und bei Männern nur wenige Daten aus prospektiven Studien", so Teich. Als generell unbedenklich könnten jedoch Mesalazin (Salofalk ${ }^{\bullet}$ ), Sulfasalazin, Budesonid, niedrig dosierte systemische Glukokortikoide, Azathioprin (Azafalk ${ }^{ø}$ ) und TNF-Blocker angesehen werden.

Nur Methotrexat (MTX) sollte in der Frühschwangerschaft vermieden werden, auch wenn die meisten Kinder, die ungewollt einer MTX-Exposition in der Embryo- und/oder Fetogenese ausgesetzt waren, gesund sind. „Der wich- tigste Risikofaktor für Mutter und Kind ist nicht die medikamentöse Therapie, sondern eine hohe Krankheitsaktivität", so Teich. Deshalb sei es wichtig, dass vor und während der Schwangerschaft eine stabile Remission bzw. eine niedrige Krankheitsaktivität erreicht werden. Auch die enge Zusammenarbeit zwischen Gynäkologe und Gastroenterologe sei unabdingbar.

Was Azathioprin bei Crohn-Patienten betrifft, so empfiehlt sich ein Einsatz aber nur bei Vorliegen von Risikofaktoren für einen komplizierten Verlauf. Patienten, die gut darauf ansprechen, müssen seltener operiert werden.

Dr. Peter Stiefelhagen

- 27. interdisziplinäres Symposium Chronisch entzündliche Darmerkrankungen, 122. Kongress der Deutschen Gesellschaft für Innere Medizin (DGIM); Mannheim, April 2016 (Veranstalter: Falk) 